\title{
Total Laparoscopic Radical Antegrade Modular Pancreatosplenectomy with Left-Posterior Superior Mesenteric Artery First-approach for Distal Pancreatic Cancer (with video)
}

Thanh Khiem Nguyen

Bach Mai Hospital

Ham Hoi Nguyen

Bach Mai Hospital

Kim Khue Dang

Bach Mai Hospital

Tuan Hiep Luong ( $\sim$ hiep1995hsgs@gmail.com )

Hanoi Medical University https://orcid.org/0000-0001-7900-9523

\section{Research Article}

Keywords: Total Laparoscopic, Radical antegrade modular pancreatosplenectomy, Left posterior SMA first-approach.

Posted Date: February 8th, 2022

DOI: https://doi.org/10.21203/rs.3.rs-1268473/v1

License: (9) This work is licensed under a Creative Commons Attribution 4.0 International License.

Read Full License 


\section{Abstract}

Pancreatic ductal adenocarcinoma (PDAC) is one of the most malignant gastrointestinal cancers. Among them, tumors of the pancreatic body and tail, or left-sided PDAC are uncommon, which accounted for about one-third of all the pancreatic neoplasms[1], and supposedly seem more aggressive than tumors of pancreatic head, not only cause by the late presentation, but also due to the difference of molecular characteristics, embryology and histology and therapeutic response[2].

Surgery is considered the only curative treatment method in pancreatic cancer, and Radical antegrade modular pancreatosplenectomy (RAMPS), which was firstly published by Strasberg et al., was proved to be superior in safe and effective aspects while comparing with standard retrograde pancreatosplenectomy (SRPS) in recent systematic literature reviews and meta-analyses[3-5]. And according to Guidelines of National Comprehensive Cancer Network (NCCN) as well as Japanese Pancreas Society (JPS), one of major factors that determined the respectability of PDAC was Superior Mesenteric Artery (SMA)'s infiltration[6, 7]. So that, SMA first-approach has been proved with benefits of improving R0-resection rate and better perioperative outcomes, as well as better long-term survival particularly[8]. Recently, with the development of laparoscopic instruments and techniques, total laparoscopic SMA first-approach RAMPS was recently proceeded by some surgeons with optimistic perioperative outcomes versus open SMA first-approach RAMPS $[9,10]$.

With experiment of total laparoscopic SMA first-approach PD, herein we present a novel technique called total laparoscopic left-posterior SMA first-approach RAMPS, with demonstrated on describing the procedure step by step with illustrative images and video.

\section{Surgical Technique}

We use 5 trocars: one $10 \mathrm{~mm}$-trocar placed through the umbilicus for camera; two $12 \mathrm{~mm}$-trocars placed at the midclavicular line higher $1 \mathrm{~cm}$ compared to umbilicus in the right and left side for instrument; two $5 \mathrm{~mm}$-trocars placed at right and left subcostal, and the patient placed in a Trendelenburg position. Surgeon stands on the right side of patient in the SMA's dissection phase, and changes to the middle position when dissecting the posterior surface of the artery with the rest of the surgical phases, the second and third assistants holding the middle and right cameras, the first assistant standing on the right side.

The retroperitoneum is opened at the left-posterior side of the mesopancreatoduodenum along to the inframeso-tranversecolon, so that the left border of the SMA's origin, the anterior surface of the aorta (AO), inferior vena cave (IVC), left renal vein (LRV), left adrenal grand (LAG), and kidney are completely exposed, as well as the posterior side of pancreatic body (Figure 1). The anterior or posterior RAMPS were performed depending on whether the tumor infiltrated to the LAG or not[3]. Then, the gastrocolic and gastrosplenic ligaments are divided, and the inferior border of the pancreas had been dissected and separated from the superior mesenteric vein (SMV) below the pancreatic isthmus, removed the lymph 
nodes (LNs) groups $14 \mathrm{v}$ and 17. Then, go upward to the superior side of the pancreas to dissect of LNs groups 7,8,9,11p,12 en bloc. After transecting the pancreas, carefully dissect the distal pancreas with the SMA, including dissection of LNs group 14p, $d$ or SMA LNs with preserving the nerve plexus around the SMA (pISMA) and celiac axis (plCE) (Figure 2). Left mesopancreas, or 'lame rétroportale gauche'[11], a retropancreatic structure connected the left-side SMA with distal pancreas was dissected en bloc with specimen (Figure 3).

\section{Results}

Our patient is a 67-year-old female with a history of diabetes and hypertension, recruited because of upper left quadrant abdominal pain. She was referred for pancreatosplenectomy because of a $3 \mathrm{~cm}$ sized mass in distal pancreas. The operation time was $240 \mathrm{~min}$, the estimated blood loss was $200 \mathrm{ml}$. With no postoperative complications as well as no diarrhea, the patient was discharged on the POD8 uneventfully. Pathological result: Pancreatic ductal adenocarcinoma with T2N1 staging and negative margin (R0).

\section{Conclusion}

Total laparoscopic left-posterior SMA first-approach RAMPS for distal pancreatic cancer is safe and feasible, with acute negative posterior margin and precise lymphadenectomy.

\section{Declarations}

Sources of funding: The authors declare no funding for this study.

Conflict of interests: The authors declare that they have no conflicts of interests.

Ethics approval: The study was approved by the Research Ethics Committee of Bach Mai Hospital. The procedures used in this study adhere to the tenets of the Declarations of Helsinki.

Acknowledgments: None.

Consent: The written informed consent was obtained from the recruited patients.

Availability of data and material: Data is available upon reasonable request and with permission of Bach Mai Hospital.

Provenance and peer review: Not commissioned, externally peer reviewed.

Guarantor: Thanh Khiem NGUYEN, M.D PhD; Ham Hoi NGUYEN, MD.

\section{Authorship contribution statement:}

Thanh Khiem NGUYEN: the co-first author and main doctor conceived the original idea and operated the patients, wrote manuscript. 
Ham Hoi NGUYEN: the co-first author and main doctor conceived the original idea and operated the patients, wrote manuscript.

Kim Khue DANG: operated the patients, summed up, revised manuscript, designed technical videos and figures.

Tuan Hiep LUONG: followed up the patients, wrote manuscript, designed technical videos and figures.

Van Duy LE: operated the patients, summed up, revised manuscript.

Duc Dung TRAN: designed technical videos and figures.

All authors contributed to the interpretation of the results, discussed the results. All authors read and approved the final manuscript to submit.

\section{References}

1. Barreto SG, Shukla PJ, Shrikhande SV: Tumors of the Pancreatic Body and Tail. World J Oncol 2010, 1(2):52-65.

2. Luo G, Jin K, Cheng H, Guo M, Gong Y, Fan Z, Yang C, Huang Q, Ni Q, Liu C et al: Prognosis of distal pancreatic cancers controlled by stage. Exp Ther Med 2020, 20(2):1091-1097.

3. Cao F, Li J, Li A, Li F: Radical antegrade modular pancreatosplenectomy versus standard procedure in the treatment of left-sided pancreatic cancer: A systemic review and meta-analysis. BMC Surg 2017, 17(1):67-67.

4. Dragomir M, Eftimie MA: Is Radical Antegrade Modular Pancreatosplenectomy the Solution? A Systematic Literature Review and Meta-Analysis. Chirurgia (Bucharest, Romania : 1990) 2017, 112(6):653-663.

5. Zhou Y, Shi B, Wu L, Si X: A systematic review of radical antegrade modular pancreatosplenectomy for adenocarcinoma of the body and tail of the pancreas. HPB 2017, 19(1):10-15.

6. Isaji S, Murata Y, Kishiwada M: New Japanese Classification of Pancreatic Cancer. In: Pancreatic Cancer. edn. Edited by Neoptolemos JP, Urrutia R, Abbruzzese JL, Büchler MW. New York, NY: Springer New York; 2018: 1021-1037.

7. Noda Y, Goshima S, Kawada H, Kawai N, Miyoshi T, Matsuo M, Bae KT: Modified National Comprehensive Cancer Network Criteria for Assessing Resectability of Pancreatic Ductal Adenocarcinoma. American Journal of Roentgenology 2018, 210(6):1252-1258.

8. Kawabata Y, Hayashi H, Takai K, Kidani A, Tajima Y: Superior mesenteric artery-first approach in radical antegrade modular pancreatosplenectomy for borderline resectable pancreatic cancer: a technique to obtain negative tangential margins. Journal of the American College of Surgeons 2015, 220(5):e49-54. 
9. Yamamoto M, Zaima M, Yamamoto H, Harada H, Kawamura J, Yamada M, Yazawa T, Kawasoe J: New laparoscopic procedure for left-sided pancreatic cancer-artery-first approach laparoscopic RAMPS using 3D technique. World Journal of Surgical Oncology 2017, 15(1):213.

10. Kawabata Y, Hayashi H, Kaji S, Fujii Y, Nishi T, Tajima Y: Laparoscopic versus open radical antegrade modular pancreatosplenectomy with artery-first approach in pancreatic cancer. Langenbeck's archives of surgery 2020, 405(5):647-656.

11. Guilloteau G, Hamel A, Hamel O, Robert R, Rogez JM, Lagier S, Blin Y, Dupas B, Le Borgne J: La lame rétro-portale droite. Morphologie 2006, 90(289):94.

\section{Figures}

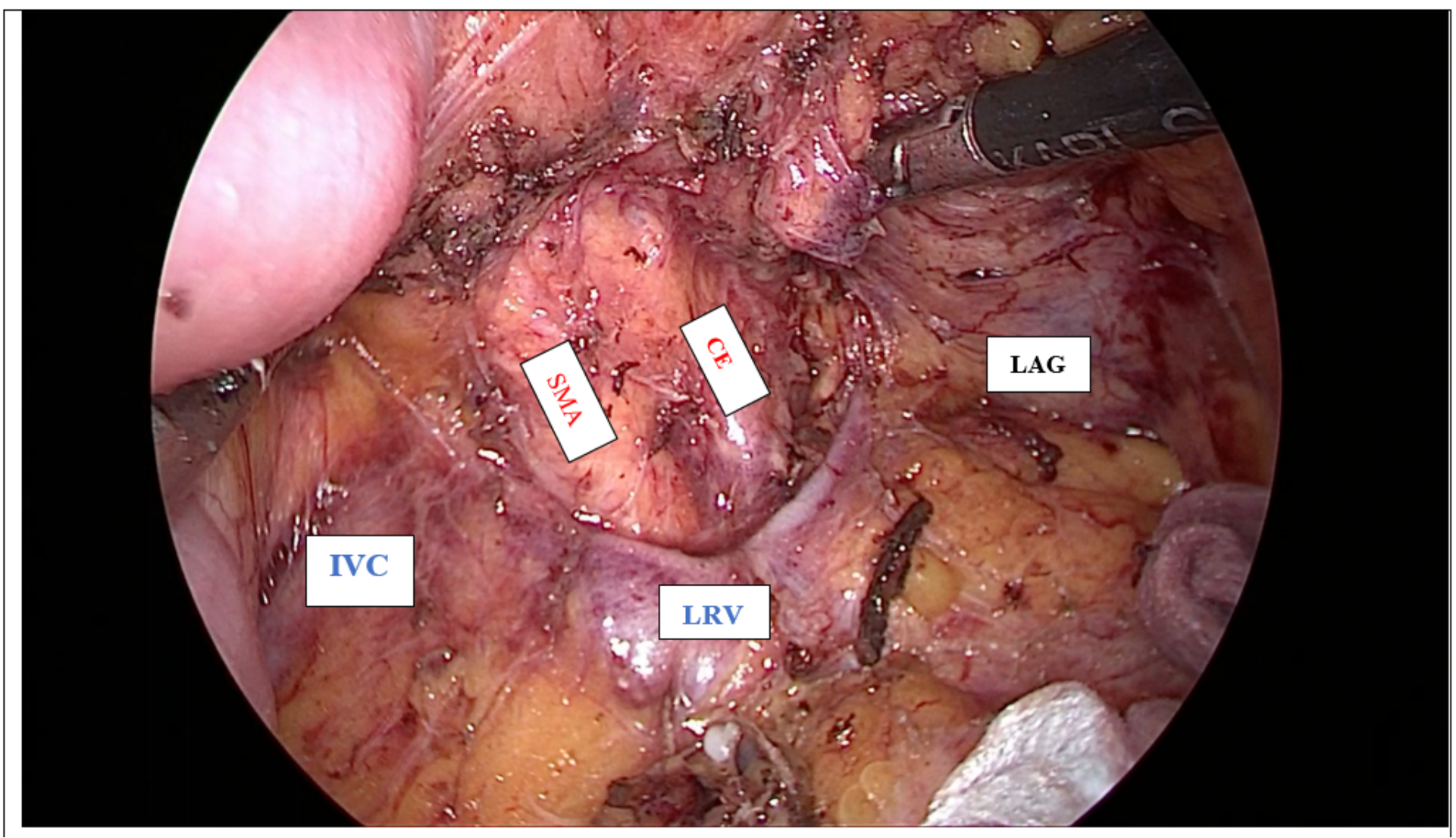

Figure 1

The retroperitoneum is opened at the left-posterior side of the mesopancreatoduodenum to excluding posterior surgical invasion

(SMA: Superior Mesenteric Artery, CE: celiac axis, IVC: inferior vena cave, LRV: left renal vein, LAG: left adrenal grand) 


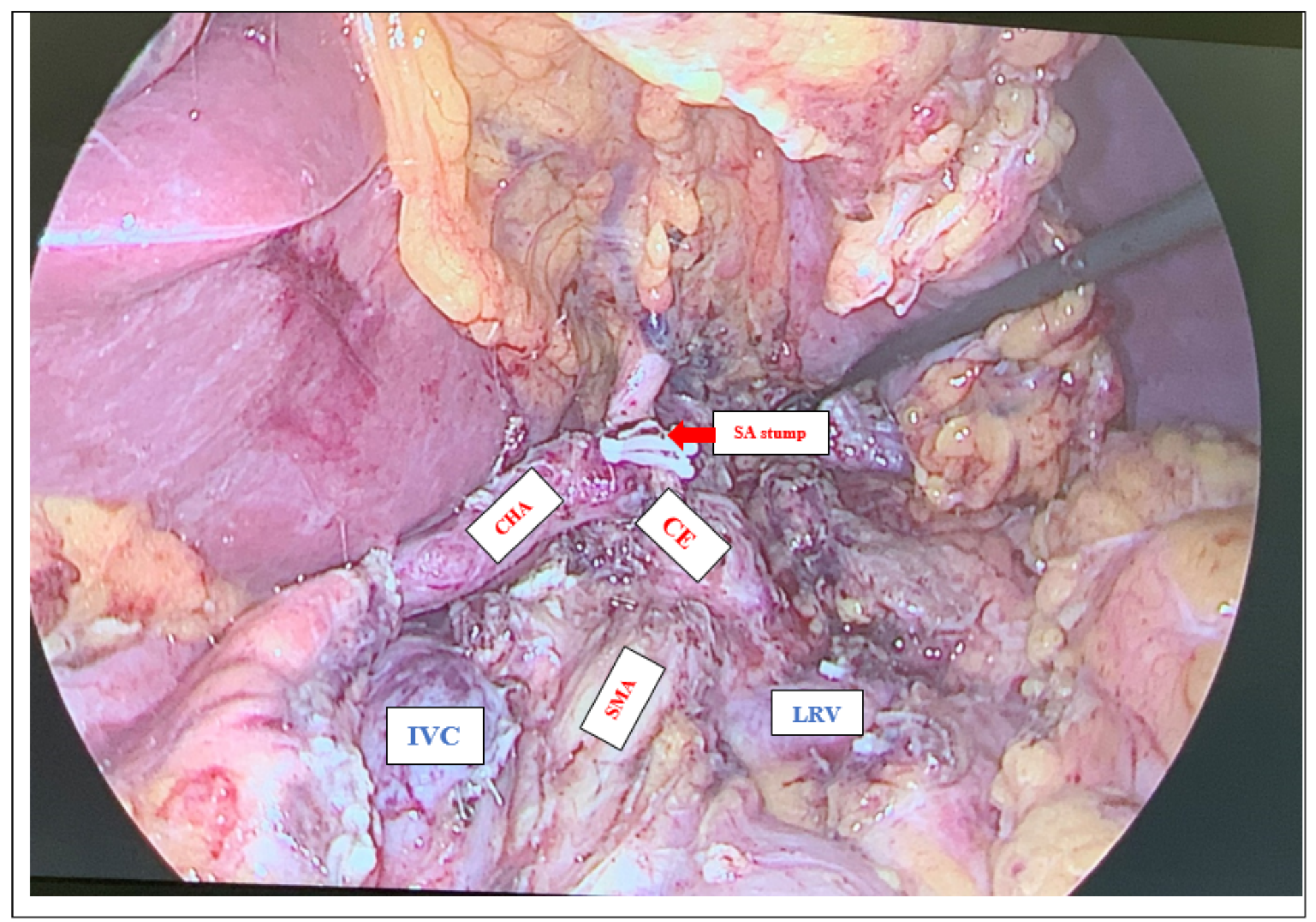

Figure 2

Dissection of LNs groups 7,8,9,11p,12 en bloc and LNs group 14p, $d$ or SMA LNs with preserving the nerve plexus around the SMA (pISMA) and celiac axis (pICE)

(SMA: Superior Mesenteric Artery, CE: celiac axis, CHA: Common hepatic artery, SA: Splenic artery, IVC: Inferior vena cave, LRV: Left renal vein) 

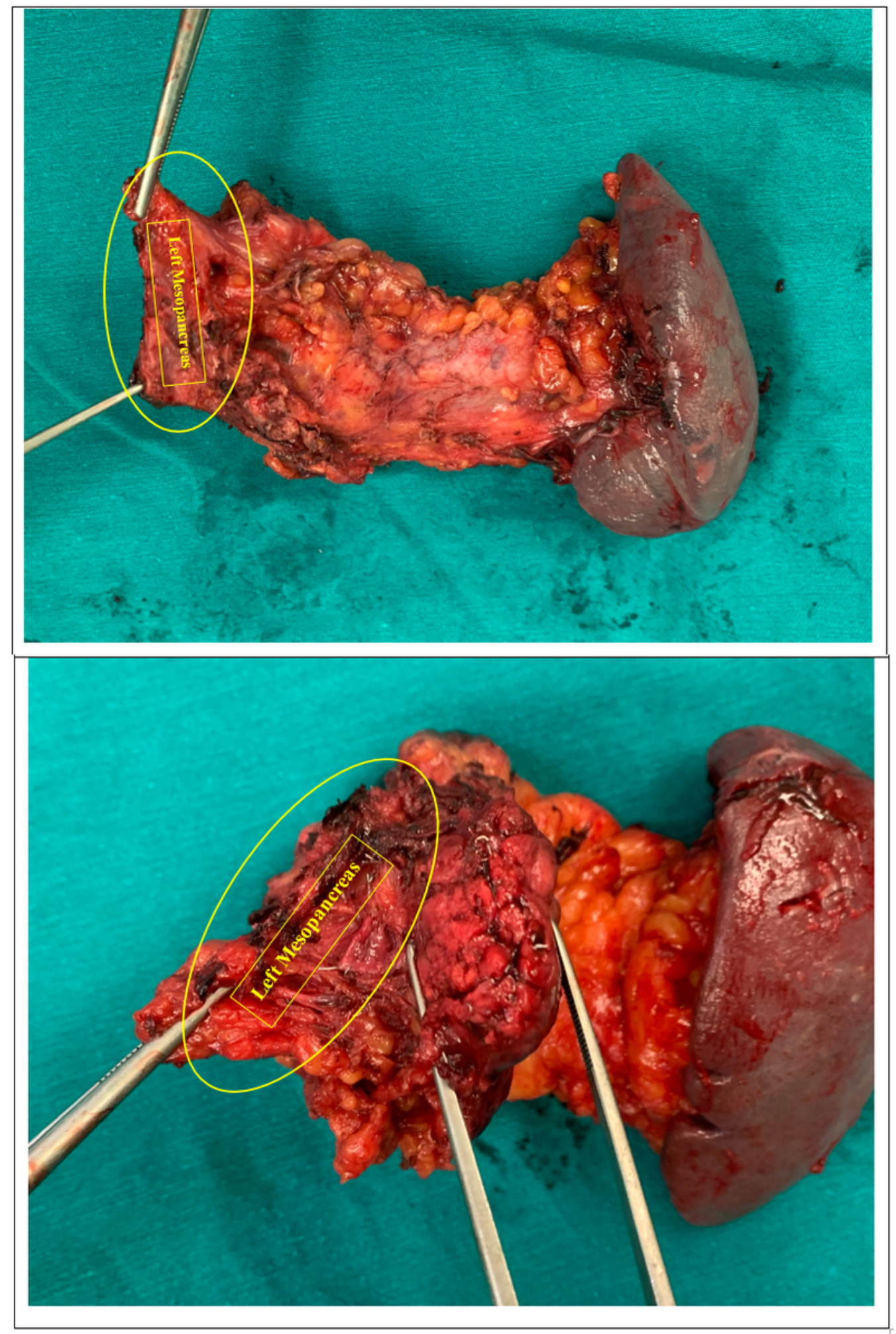

Figure 3

Specimen with the left mesopancreas, or 'lame

rétroportale gauche'. 


\section{Supplementary Files}

This is a list of supplementary files associated with this preprint. Click to download.

- Video1.mp4 